\title{
High-resolution intravascular magnetic resonance quantification of atherosclerotic plaque at 3T
}

Di Qian ${ }^{1}$ and Paul A Bottomley ${ }^{1,2^{*}}$

\begin{abstract}
Background: The thickness of fibrous caps (FCT) of atherosclerotic lesions is a critical factor affecting plaque vulnerability to rupture. This study tests whether 3 Tesla high-resolution intravascular cardiovascular magnetic resonance (CMR) employing tiny loopless detectors can identify lesions and accurately measure FCT in human arterial specimens, and whether such an approach is feasible in vivo using animal models.
\end{abstract}

Methods: Receive-only $2.2 \mathrm{~mm}$ and $0.8 \mathrm{~mm}$ diameter intravascular loopless CMR detectors were fabricated for a clinical 3 Tesla MR scanner, and the absolute signal-to-noise ratio determined. The detectors were applied in a twostep protocol comprised of CMR angiography to identify atherosclerotic lesions, followed by high-resolution CMR to characterize FCT, lesion size, and/or vessel wall thickness. The protocol was applied in fresh human iliac and carotid artery specimens in a human-equivalent saline bath. Mean FCT measured by $80 \mu \mathrm{m}$ intravascular CMR was compared with histology of the same sections. In vivo studies compared aortic wall thickness and plaque size in healthy and hyperlipidemic rabbit models, with post-mortem histology.

Results: Histology confirmed plaques in human specimens, with calcifications appearing as signal voids. Mean FCT agreed with histological measurements within $13 \%$ on average (correlation coefficient, $R=0.98$; Bland-Altman analysis, $-1.3 \pm 68.9 \mu \mathrm{m})$. In vivo aortic wall and plaque size measured by $80 \mu \mathrm{m}$ intravascular CMR agreed with histology.

Conclusion: Intravascular 3T CMR with loopless detectors can both locate atherosclerotic lesions, and accurately measure FCT at high-resolution in a strategy that appears feasible in vivo. The approach shows promise for quantifying vulnerable plaque for evaluating experimental therapies.

Keywords: Interventional MR, Intravascular MR, Atherosclerosis, Fibrous cap thickness, High-resolution

\section{Background}

Atherosclerosis is characterized in its advanced stages by lesions containing extra-cellular lipids, foam cells and/or calcium deposits in the arterial wall, covered by fibrous caps [1-3]. Its clinical symptoms often involve progressive myocardial, cerebral or peripheral ischemia resulting from the ensuing stenosis, but lesion rupture can cause acute thrombosis, stroke, heart attack and death [1-5]. Fibrous cap thickness (FCT) is an important factor in determining the vulnerability of atherosclerotic plaques. Because thin caps are associated with large plaque stress and the likelihood of rupture [3-6], an ability to accurately quantify

\footnotetext{
* Correspondence: bottoml@mri.jhu.edu

'Division of MR Research, Department of Radiology and Radiological

Science, Johns Hopkins University, Baltimore, MD, USA

Full list of author information is available at the end of the article
}

FCT in situ could prove key to identifying plaques that are most vulnerable to rupture and would benefit most from intervention, as well as evaluating the mechanistic and morphological changes responsive to therapies aimed at plaque regression.

While an FCT of less than $65 \mu \mathrm{m}$ has been used to classify fibrous caps in coronary plaques as vulnerable [7-10], currently there isn't an accepted clinical methodology for identifying unstable plaques in patients in situ [11]. X-ray angiography routinely detects stenoses by imaging the arterial lumen, but not the vessel wall or lesion morphology per se, nor early-stage lesions with mild-to-moderate stenoses [5]. Intravascular ultrasound (IVUS) can provide trans-luminal imaging at $\geq 100 \mu \mathrm{m}$ resolution without ionizing radiation $[9,11,12]$, although it is typically performed during withdrawal of an imaging

\section{() Biomed Central}


catheter threaded over a guidewire inserted under X-ray fluoroscopy [13]. IVUS studies of human endarterectomy specimens suggest that an FCT threshold of $650 \mu \mathrm{m}$ can discriminate symptomatic from asymptomatic carotid artery stenoses [14]. Optical coherence tomography (OCT) is another intravascular imaging option [15] that is fast, has high resolution $(10-20 \mu \mathrm{m})$, but a limited penetration depth of about $1 \mathrm{~mm}[9,10,15,16]$. It also requires a blood-free environment, afforded by intermittent saline flushes through an X-ray fluoroscopic guide catheter $[9,10]$.

Cardiovascular magnetic resonance (CMR) offers the multi-functionality and intrinsically high soft-tissue contrast that could potentially add unique value and provide intravascular imaging without X-ray guidance or ionizing radiation [17-20]. However, when applied noninvasively with external detector coils, CMR's spatial resolution for differentiating FCT is more limited $(>200 \mu \mathrm{m})$ than both IVUS and OCT [17-20]. To improve local sensitivity and spatial resolution, intravascular CMR employing tiny detectors as guidewires or catheters was proposed many years ago [21-26]. At clinical field strengths of $\mathrm{B}_{0}=1.5$ Tesla ( $\mathrm{T})$, these devices have demonstrated efficacy in characterizing vessel wall thickness and plaque components in larger vessels with in-plane resolution $\geq 100 \mu \mathrm{m}$ [27-30]. Nevertheless, as emphasized in recent editorials, the speed and spatial resolution of intravascular CMR remain a challenge [31,32]. A resolution of $80 \mu \mathrm{m}$ or better may be needed to resolve plaque components in smaller critical vessels, and at this time it is not known whether an intravascular CMR modality will ever be a practical tool for plaque characterization or FCT measurements in patients [31].

Yet the recent emergence of clinical 3T MR scanners does afford some new opportunity for addressing these problems by virtue of an almost quadratic gain in signalto-noise ratio (SNR) with $\mathrm{B}_{0}$, due to the intrinsic noise properties of tiny detectors $[33,34]$. As a consequence, "loopless antenna" detectors [25] realizing a $~ 4$-fold higher SNR and a 10 -fold increase in visual field-of-view (FOV) at $3 \mathrm{~T}$ compared to the original $1.5 \mathrm{~T}$ detectors, have been demonstrated [33]. This, with local heating controlled at $\leq 1^{\circ} \mathrm{C}$ [33]. In the present study, we investigate whether such 1-2 mm diameter detect-only $3 \mathrm{~T}$ intravascular antenna guidewires can both identify atherosclerotic lesions without X-ray fluoroscopic guidance, and provide high-resolution $(<100 \mu \mathrm{m})$ imaging of vessel anatomy and accurate FCT and wall thickness measurements in a conventional clinical 3T MR scanner. FCT is measured at $80 \mu \mathrm{m}$ resolution in diseased human arterial specimens in vitro, and the results compared with histology. The feasibility of the approach is then demonstrated in vivo in healthy and hyperlipidemic rabbit aortas, a vessel that is comparable in size to a human coronary.

\section{Methods}

Devices

In vitro $\mathrm{CMR}$ studies were performed with a $3 \mathrm{~T}$ loopless "receive-only" detector fabricated from a UT-85C (outer diameter, $\mathrm{OD}=2.2 \mathrm{~mm}$ ) semi-rigid copper coaxial cable (Micro-coax, Inc., Pottstown, PA) to fit within the approximately $8-15 \mathrm{~mm}$ lumens of human vessel specimens studied (Figure 1). The length of the detector's coaxial cable was tuned to a quarter-wavelength $(\lambda / 4 \approx$ $40 \mathrm{~cm})$. The central conductor was extended an additional $4 \mathrm{~cm}$ beyond the cable portion to form a whip that maximized signal at the whip-cable junction [33]. The outer surface of the cable was insulated with $0.02 \mathrm{~mm}$ polyester (dielectric constant, $\varepsilon=3$ ) heat-shrink tubing (Advanced Polymer Inc, Salem, NH). To determine its sensitivity in the plane of the cable for angiography and/ or tracking, the absolute SNR of the device in a $0.35 \%$

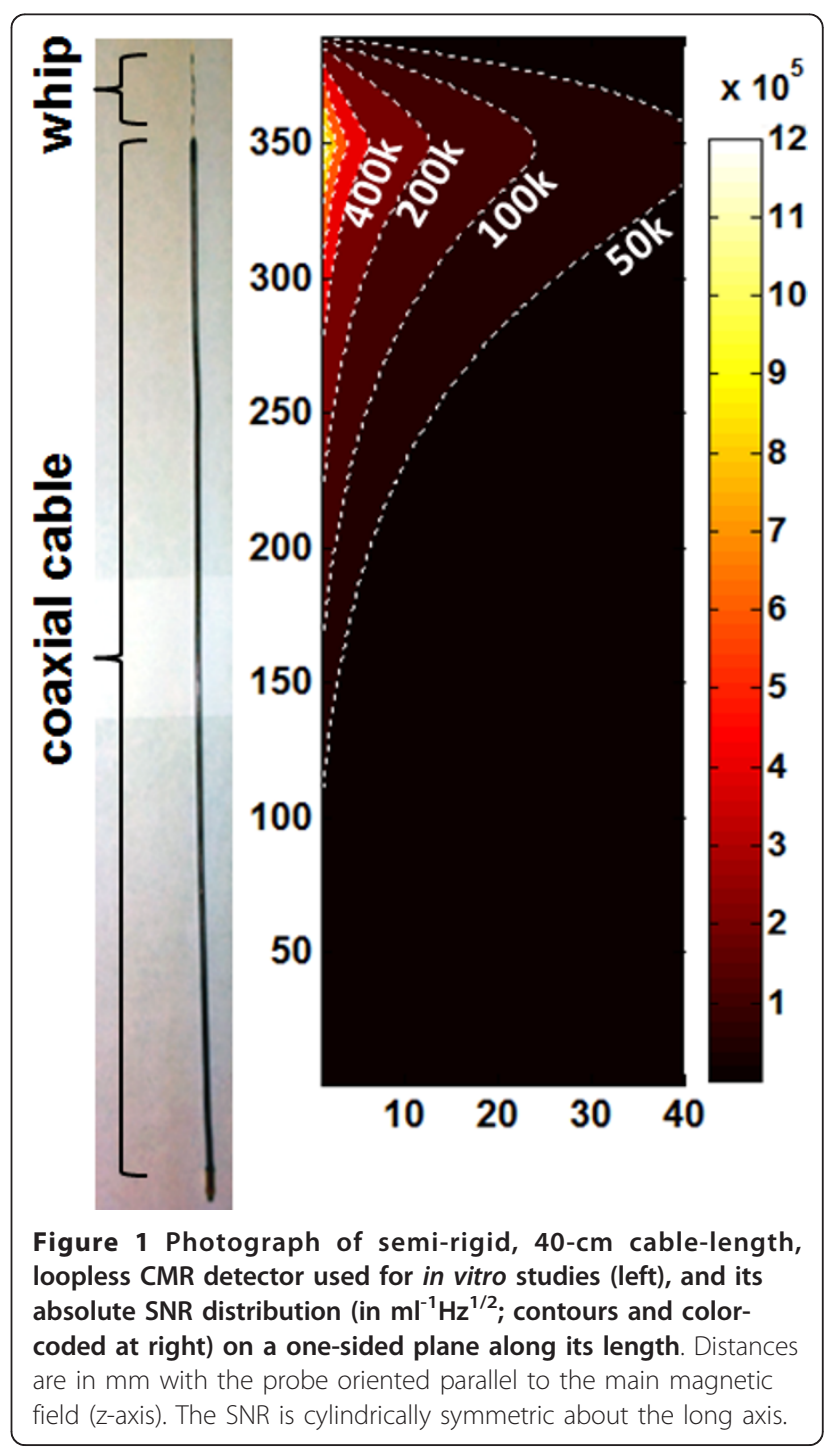


saline phantom with radio-frequency $(\mathrm{RF})$ electrical properties comparable to those of tissue (conductivity, $\sigma=0.6$ $\mathrm{S} / \mathrm{m}$, dielectric constant $\varepsilon=80$ ) was determined by the numerical method-of-moments (FEKO EM analysis software, Stellenbosch, South Africa) from the ratio of the circularly polarized RF field excited by a $1 \mathrm{~A}$ current, to the square root of the antenna resistance in the saline [33]. Since the SNR is cylindrically symmetric about the cable's long axis, the computation was performed on a single plane on one side of the detector, over the range $0.1 \leq r \leq 40 \mathrm{~mm}$ from the detector outer conductor, and $0 \leq z \leq 39 \mathrm{~cm}$ from the detector tip, as well as in axial planes about the detector junction.

In vivo experiments were performed with a biocompatible nitinol loopless imaging detector (Figure 2) with the same coaxial structure as the detector used for the in vitro experiments. It was formed by modifying an obsolete $0.76 \mathrm{~mm}$ OD Intercept 1.5T guidewire (MRI Interventions, formerly Surgi-Vision Inc, Memphis TN) for 3T use by retuning the whip to $40 \mathrm{~mm}$, and extending the cable length to $3 \lambda / 4$ (for a total cable length of $120 \mathrm{~cm}$ ) to permit practical in vivo use in the magnet bore. Device heating during CMR was controlled at $\leq 1^{\circ} \mathrm{C}$ with decoupling circuitry described previously, and demonstrated in safety studies on a saline gel phantom [33]. The detector was tuned, matched, and interfaced to a 3T Philips Achieva MR scanner equipped with an X-ray C-arm (Philips Healthcare, Cleveland, $\mathrm{OH}$ ) [33].

\section{In vitro Studies}

The 3T loopless CMR detector was first used to identify suspected plaques inside a fresh $\sim 10 \mathrm{~cm}$-long human

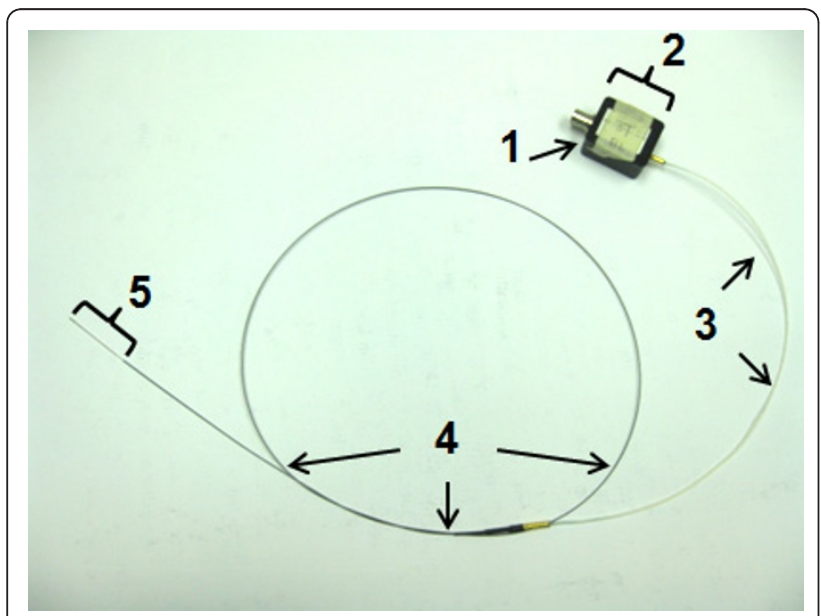

Figure 2 Photograph of the biocompatible 3T loopless CMR detector. The components are: (1) BNC connection to the MR scanner interface; (2) tuning, matching, and decoupling circuit box; (3) coaxial extension cable; (4) coaxial cable from an obsolete 1.5T Intercept guidewire $(\mathrm{OD}=0.76 \mathrm{~mm})$; and (5) the extended inner conductor whip (length $=4 \mathrm{~cm}$ ). iliac artery specimen, complete with bifurcation, harvested at autopsy within 3 hours of death. All studies of decedent specimens were approved by this institution's Office of Human Subject Research, Institutional Review Board. The vessel was secured to a plastic frame inside a $0.35 \%$ saline phantom (with $\sigma=0.6 \mathrm{~S} / \mathrm{m}, \varepsilon=80$ ). The $3 \mathrm{~T}$ detector was inserted into the lumen, and a spin-lattice relaxation time $\left(\mathrm{T}_{1}\right)$-weighted angiographic scan performed in the coronal plane along the luminal longaxis (three-dimensional fast field echo, 3D FFE; FOV = $120 \times 73 \mathrm{~mm}$; resolution, $0.5 \times 0.5 \times 1 \mathrm{~mm}$; array size = $256 \times 256 \times 10$; repetition time/echo time, $\mathrm{TR} / \mathrm{TE}=25 /$ $2.6 \mathrm{~ms}$; flip-angle, $\mathrm{FA}=20^{\circ}$; scan time $=143 \mathrm{~s}$ ). After locating suspected plaques on the coronal angiographic scans, high-resolution trans-axial spin-spin relaxation time $\left(\mathrm{T}_{2}\right)$-weighted scanning of the vessel wall was performed (3D turbo spin-echo, TSE factor $=9$; FOV $=20 \times 20 \mathrm{~mm}$; $\mathrm{TR} / \mathrm{TE}=1500 / 32 \mathrm{~ms}$; resolution $=80 \mu \mathrm{m} \times 80 \mu \mathrm{m} \times$ $2 \mathrm{~mm}$; FA $=90^{\circ} ; 4$ averages; scan time $=1514 \mathrm{~s}$ ) to reveal the fibrous cap. To determine the effect of image resolution on measurements of FCT, the same section of a human iliac specimen was imaged with the same sequence but at 80,100 and $140 \mu \mathrm{m}$ in-plane resolution.

The locations of the high-resolution trans-axial images were registered to the vessels by first measuring their distance to the ends of the vessels as visualized on the coronal images. After CMR, vessels were decalcified for $48 \mathrm{hrs}$ and cut into 5-10 mm sections, each facing the location of the corresponding trans-axial image to the nearest millimeter. The histological sections were visually inspected for antenna-related injury to the vessel wall, embedded in wax, microtomed, and stained with hematoxylin and eosin (H\&E) at this institution's pathology service. The sections were then photographed with a microscope-mounted digital camera.

To test whether high-resolution 3T interventional CMR with a loopless detector could be used to provide accurate measures of FCT, thirty-one diseased carotid artery segments with atherosclerotic lesions identified by palpation, were harvested from sixteen adult human cadavers. These vessel segments were fixed in $10 \%$ formalin at $3^{\circ} \mathrm{C}$ for $24 \mathrm{hr}$. High resolution $\mathrm{T}_{2}$-weighted $\mathrm{CMR}$ of the vessel wall was performed on vessels mounted in the saline phantom, followed by sectioning and histology as described above.

The FCT was measured in both the CMR and the histology sections between the two shoulder ends of the fibrous cap at an interval of $480 \mu \mathrm{m}$, or at about every 6 pixels in the $80 \mu \mathrm{m}$ CMR. Distance was measured in histology sections using a movable scale overlaid on the digital micrograph, and in CMR by counting pixels and multiplying by the resolution. Measurements were restricted only to the regions with clear fibrous cap and lumen borders. The average of all of the FCT measurements from each image 
was recorded as the mean FCT for that image section. The mean FCT for the iliac specimen imaged at 80, 100 and $140 \mu \mathrm{m}$ resolution was measured the same way.

\section{In vivo Studies}

High-resolution intravascular CMR was performed in vivo using the $3 \mathrm{~T}$ biocompatible loopless detector in a healthy adult New Zealand white rabbit, and a 30-month old male Watanabe heritable hyper-lipidemic (WHHL) rabbit which is prone to spontaneously develop atherosclerotic lesions. The study was approved by this institution's Animal Care and Use Committee. The animals were sedated and positioned supine in the $3 \mathrm{~T}$ scanner, and the detector inserted into the aorta via the femoral artery and a 5-French introducer under CMR guidance. The same $\mathrm{T}_{1}$-weighted angiographic coronal scan as in the in vitro studies was used, except that the scans were cardiac-triggered. The location of the detector's junction in the animal relative to the renal bifurcation was determined by $\mathrm{X}$-ray fluoroscopy and on the coronal $\mathrm{T}_{1}$ angiographic images. The locations of the high-resolution trans-axial images relative to the detector's junction and to the center of the renal bifurcation were noted for subsequent co-registration.

High-resolution trans-axial CMR of the aortic wall was performed above the renal bifurcation. $\mathrm{A}_{2}$-weighted TSE sequence modified for black-blood with cardiac gating was used for the healthy rabbit $(\mathrm{FOV}=80 \times 40 \mathrm{~mm}$; TR/TE $=$ $3000 / 21 \mathrm{~ms}$; resolution $=80 \mu \mathrm{m} \times 80 \mu \mathrm{m} \times 3 \mathrm{~mm}$; array size $=1008 \times 1008 ; \mathrm{FA}: 90^{\circ} ; 4$ averages; scan time $=597 \mathrm{~s}$, and a $3 \mathrm{D} \mathrm{T}_{1}$ weighted TSE black-blood sequence with shortened scan-time was used for the hyperlipidemic rabbit $(\mathrm{FOV}=20 \times 10 \mathrm{~mm}, \mathrm{TR} / \mathrm{TE}=429 / 26$; resolution $=80 \mu \mathrm{m}$ $\times 80 \mu \mathrm{m} \times 2 \mathrm{~mm}$; array size $=256 \times 256 \times 10 ;$ FA: $90^{\circ}$; 8 averages; scan time $=436 \mathrm{~s}$ ). After CMR, the rabbits were humanely sacrificed. The aortas were harvested and cut into $5 \mathrm{~mm}$ sections facing the locations at which transaxial CMR was performed, referenced relative to the renal bifurcation. The sections were visually inspected for injury, then H\&E stained for histology as above.

\section{Results}

\section{In vitro Studies}

The computed absolute SNR distribution of the loopless detector is shown in Figure 1. The highest SNR occurs at the detector's whip-cable junction at a radial distance $r=$ $0.1 \mathrm{~mm}$, falling to $17 \%$ of the peak at $r=5 \mathrm{~mm}$. However, excluding the tip [35], the absolute SNR of the device for small $r$ is preserved to at least half of the peak value over an extended region in the longitudinal plane for distances $z \leq 80 \mathrm{~mm}$ from the device junction.

Figure 3 exemplifies $T_{1}$-weighted angiographic scout CMR, and high-resolution trans-axial images acquired from human iliac artery specimens in vitro. In coronal angiographic images, calcified plaques appeared as regions of low intensity or signal voids, such as those between the vessel walls below the iliac bifurcation in Figure 3(A). The guide-wire CMR detector itself contains no mobile protons and also appears as a signal void: the absence of any image decoupling artifact around the probe attests to the efficacy of the tune/ match/decoupling circuitry $[33,35]$. The extended region of sensitivity permits high-resolution imaging virtually anywhere in these lesions, and the trans-axial $80 \mu \mathrm{m}$ image of the iliac vessel wall (Figure 3B) reveals a calcified plaque as a void separated from the fluid-filled lumen by the fibrous cap. The vessel wall morphology is confirmed by microscopy of the corresponding histological section (Figure 3C).

CMR of a human iliac vessel acquired at 80,100 , and $140 \mu \mathrm{m}$ resolution is presented in Figure 4 with corresponding histology. Mean FCT was 360, 243, and 206 $\mu \mathrm{m}$, when measured with 140,100 , and $80 \mu \mathrm{m}$ resolution intravascular CMR, respectively (Figure 4A-C). Mean FCT from histology was lower at $186 \mu \mathrm{m}$ (Figure 4D), consistent with the view that reducing the CMR spatial resolution (increasing voxel size) decreases the accuracy of FCT measurements.

All 31 vessel segments exhibited lesions with fibrous caps based on histology. High-resolution CMR of carotid vessel walls at $80 \mu \mathrm{m}$ also revealed atheroma in all vessel segments, permitting 3-13 individual FCT measurements from each CMR section, and 3-12 FCT measurements in each of the corresponding histological sections, from which the mean FCT was determined. The mean FCT measured from CMR is plotted against the mean FCT from histology in Figure 5. The mean FCT by histology ranged from $74 \mu \mathrm{m}$ to $1.1 \mathrm{~mm}$. The two sets of measurements are highly correlated (correlation coefficient, $\mathrm{R}=$ 0.96). Even limiting the data to those samples with mean FCT $<650 \mu \mathrm{m}$ (24 out of 31 samples) as measured by histology, preserves the correlation $(\mathrm{y}=0.921 \mathrm{x}+29.8, \mathrm{R}=$ 0.91, $\mathrm{P}=0.98$ ). Bland-Altman analysis (Figure 6) shows excellent agreement between the FCT measured by CMR and that measured by histology $(-1.3 \pm 68.9 \mu \mathrm{m})$.

\section{In vivo Studies}

The placement of a biocompatible 3T loopless detector in the New Zealand White rabbit aorta in vivo under CMR angiography is exemplified in Figure 7(A). This shows the detector's whip-cable junction where signal intensity is highest (Figure 1), $4 \mathrm{~cm}$ above the renal bifurcation. Unlike CMR endoscopic probes [33,34], the receive-only intravascular detector used here reveals the entire aorta and some adjacent anatomical structures, including the renal bifurcation. X-ray fluoroscopy confirmed the location of the whip-cable junction $4 \mathrm{~cm}$ above the renal-bifurcation (Figure $7 \mathrm{~B})$. An $80 \mu \mathrm{m}$ trans-axial high-resolution CMR of the 


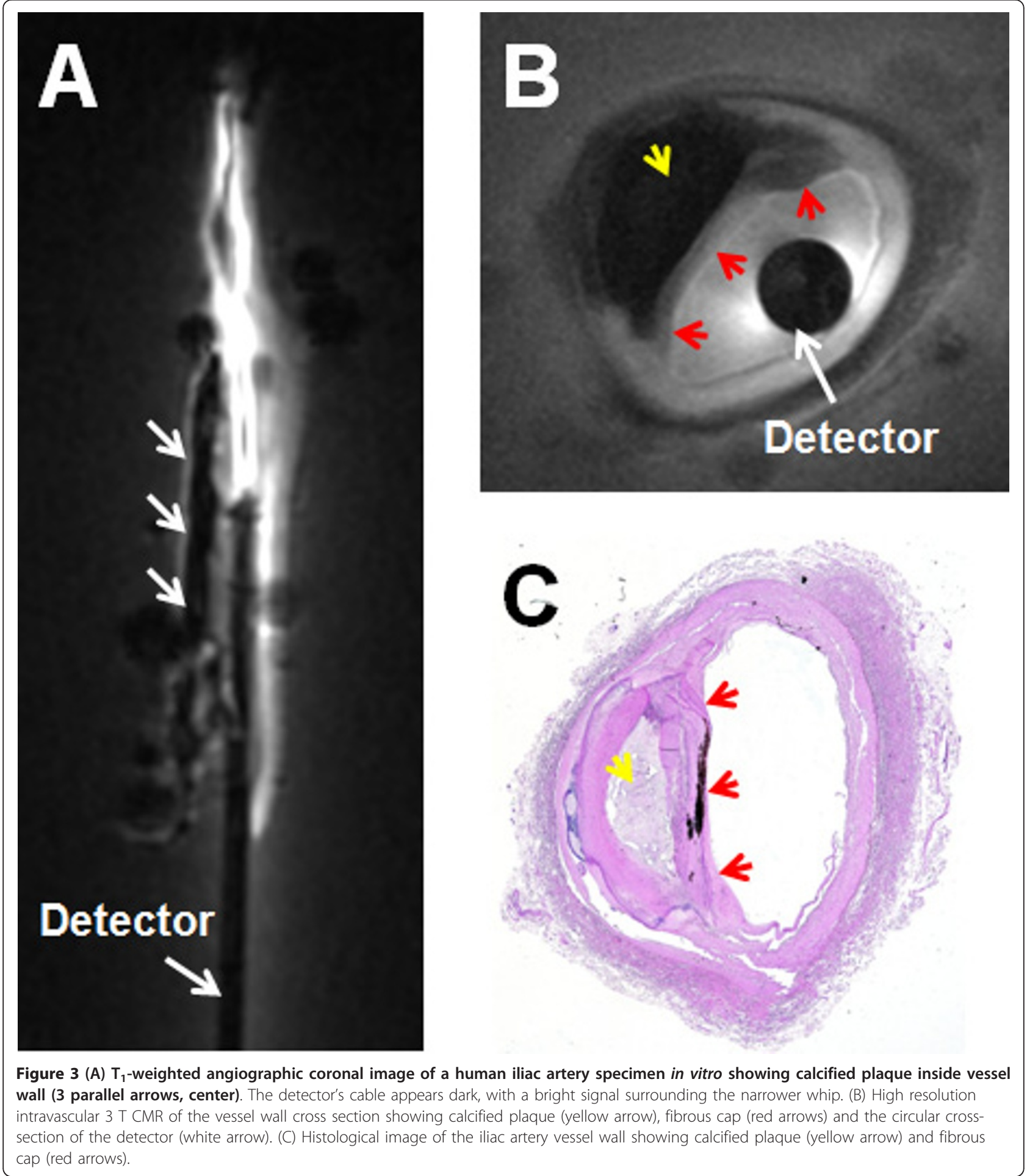

aorta delineates the vessel wall in the rabbit (Figure 7C). The vessel wall thickness was measured from the image at 12 equi-angular locations around the vessel, commencing at a vessel feature identifiable on both CMR and histological images (solid arrows, Figure 7C, D). The average wall thickness from high-resolution intravascular 3T CMR was
$0.54 \mathrm{~mm} \pm 0.06(\mathrm{SD})$ (Figure $7 \mathrm{C}$ ), in agreement with an average thickness of $0.55 \pm 0.07 \mathrm{~mm}$ measured the same way in the histological section (Figure 7D).

In the hyperlipidemic rabbit, in vivo $80 \mu \mathrm{m}$ high-resolution $\mathrm{T}_{1}$-weighted $\mathrm{CMR}$ revealed a region of elevated signal intensity on the inner wall of the vessel extending 


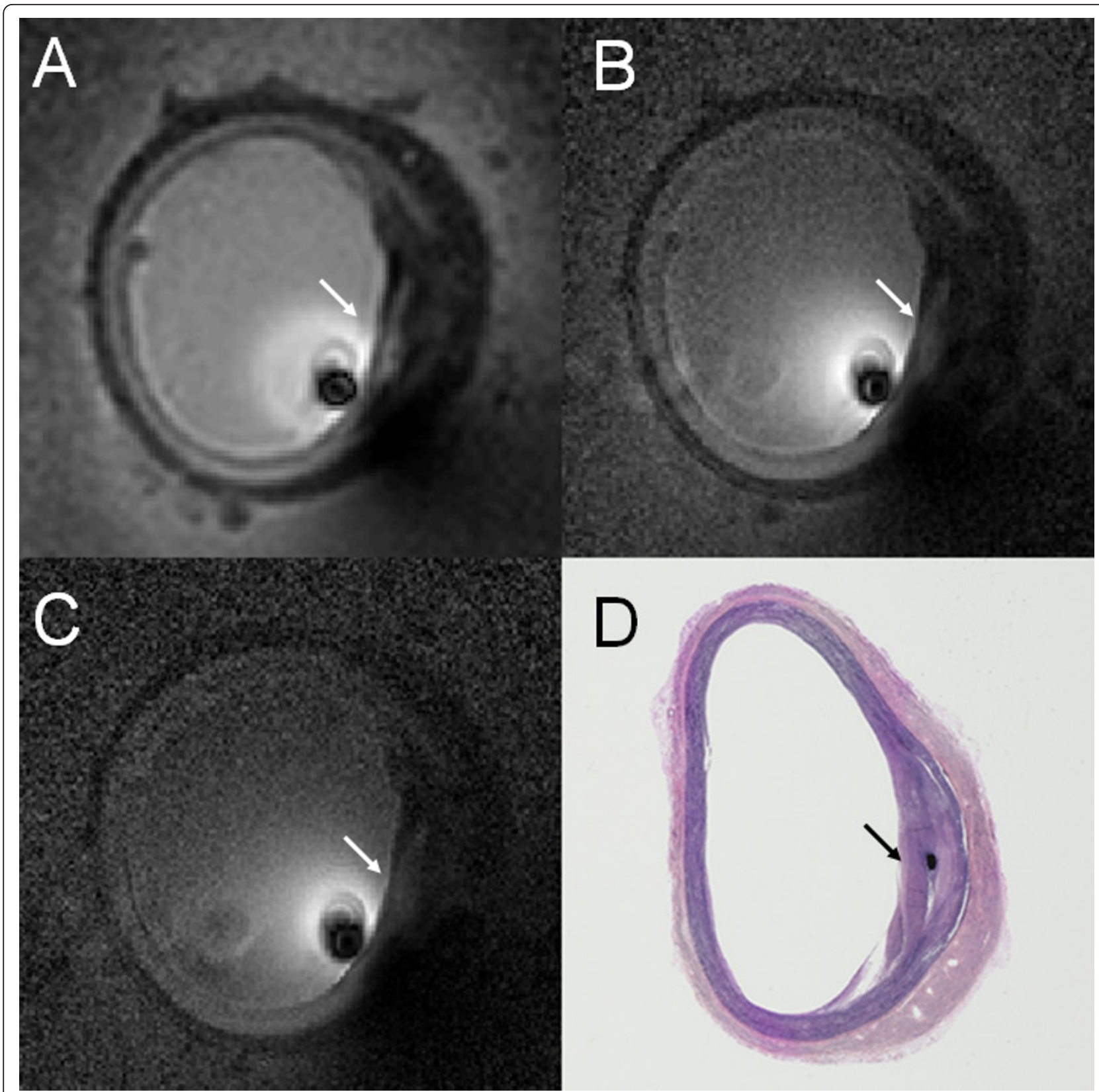

Figure 4 High resolution CMR of human iliac artery specimen acquired using the 3 T loopless CMR detector showing a fibrous cap (arrows) at (A) $140 \mu \mathrm{m}$; (B) $100 \mu \mathrm{m}$; and (C) $80 \mu \mathrm{m}$ resolution (here, image pixel intensity is inversely scaled to compensate for the $\sim 1 / r$ signal drop with distance, $r$, from the detector). The corresponding histological section is shown in (D).

into the lumen (Figure 8A, B), consistent with a pattern of fibrous atheroma tissue [29]. A small atherosclerotic lesion was confirmed in the corresponding histological section from the same location (Figure 8C). This shows a fairly homogeneous layer of fibrous tissue, with a higher lipid content rendering it paler than the adventitia. The area of the plaque region measured from CMR (Figure $8 \mathrm{~B}$ ) is $0.36 \mathrm{~mm}^{2}$, while the plaque area in the histological section is $0.33 \mathrm{~mm}^{2}$.
Visual inspection of the $5-10 \mathrm{~mm}$ sections harvested from the human vessel specimens and the rabbits prior to fixation revealed no evidence of vessel perforation, heat injury or plaque rupture attributable to the intravascular CMR detector.

\section{Discussion}

In this paper we evaluated the utility of a high-resolution $(80-\mu \mathrm{m})$ receive-only intravascular loopless CMR 


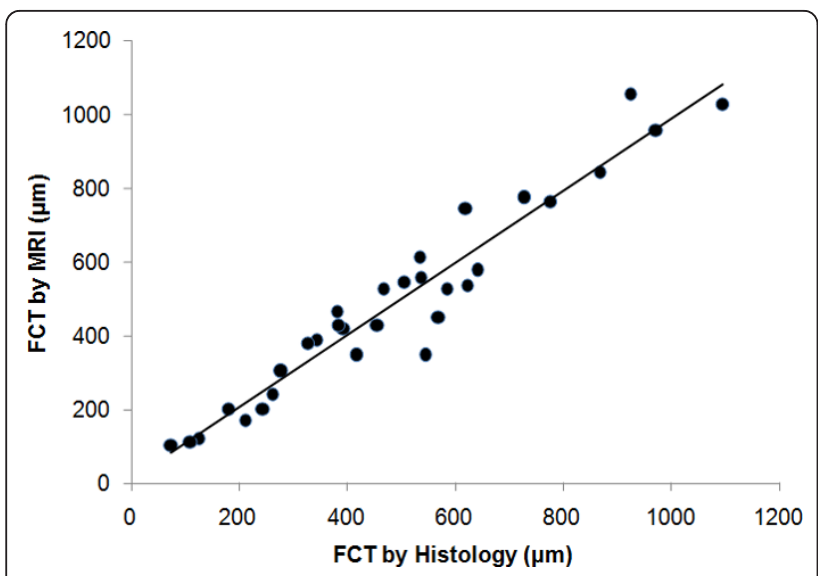

Figure 5 Comparison of mean FCT measured by highresolution intravascular $3 \mathrm{~T}$ CMR and by histology. The line of best fit for all the data $(n=31)$ is $y=0.98 x+11.5 \mu \mathrm{m}$ (correlation coefficient, $R=0.96$; probability that difference in $\mathrm{FCT}$ is insignificant, $P=0.99$ )

detector as a possible guidewire for identifying imaging and measuring the fibrous cap thickness of atherosclerotic plaques in a widely-available clinical 3T MR system. In a $1-\mathrm{cm}$ region $(r \leq 5 \mathrm{~mm})$, potentially relevant to most cases of coronary and carotid vessel imaging, the detector retains over $17 \%$ of the peak sensitivity at its junction. Moreover its extended spatial sensitivity along a lead length of about double the whip length (Figures $1,2,6)$, permits angiographic imaging using relatively low-resolution coronal CMR to identify suspected atherosclerotic lesions, and supports a guidewire function under CMR alone that does not require $\mathrm{X}$-ray fluoroscopy. This strategy differs from the CMR endoscopy approach $[36,37]$ where the detector's intrinsic sensitivity is limited to a thin sensitive disk by design, and the CMR's frame-of-reference is locked to the probe-head to provide a "probe's-eye view". Here we have shown

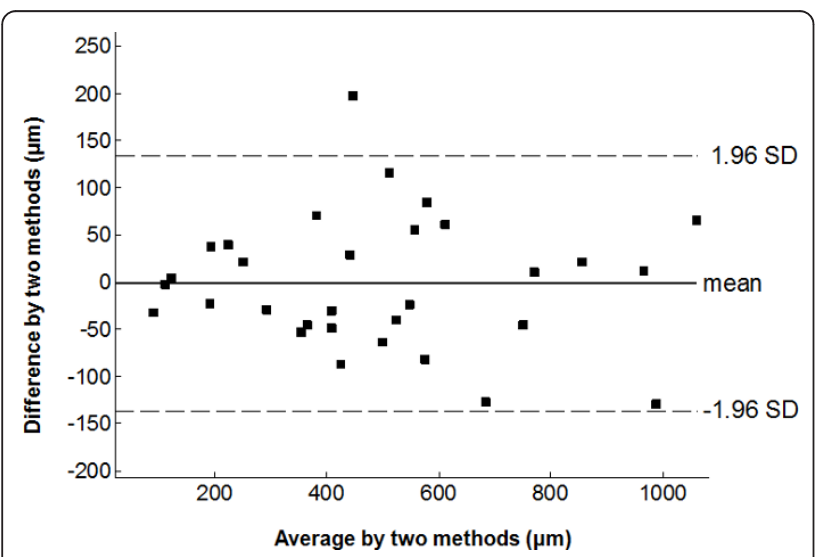

Figure 6 Bland-Altman plot for the mean FCT measured by high-resolution CMR and by histology. that, after detection by low-resolution angiographic-type CMR, suspect lesions can subsequently be targeted for high-resolution CMR that visualizes calcifications as signal voids (Figure 3 ) and permits accurate characterization of FCT (Figures 5, 6), a key predictor of plaque vulnerability to rupture.

Although the FCT measurements were only performed on human specimens in an electrically bio-analogous saline phantom, the feasibility of the approach was demonstrated with a $0.76 \mathrm{~mm}$ maximum diameter biocompatible nitinol loopless antenna guidewire in vivo in the rabbit aorta (Figures 7, 8). In these studies, artifacts from respiratory and cardiac motion were relatively minor, perhaps reflecting the limited spatial extent of high-resolution imaging. At least motion did not seem to affect the accuracy of measurements of aortic wall thickness or plaque size in $\mathrm{T}_{2}$ or $\mathrm{T}_{1}$-weighted CMR, which agreed with histological measurements to within $2 \%$ and $10 \%$, respectively. The scan time for 3D cardiac-gated in vivo $80 \mu \mathrm{m}$ high-resolution CMR with the 3T loopless detector was 7-10 min (Figures 7,8 ), which is within a range that is potentially tolerable for clinical research studies.

The improved performance of the 3T intra-vascular loopless detector compared to prior 1.5T devices derives from an approximately quadratic SNR performance with MR field-strength [33]. Here, the SNR gain was exchanged for a high-resolution intravascular imaging capability, and a higher-speed lower-resolution angiographic-type CMR that permitted device tracking. In addition, the extended CMR-sensitivity of the guidewire about the cable/whip junction obviated the need for precise device positioning or repeated tracking-scans, enabling high-resolution imaging anywhere within the detector's sensitive region. At 80 $\mu \mathrm{m}$ resolution, the $3 \mathrm{~T}$ loopless detector provided exceptional views of the vessel wall and pathology (Figures. 3, 4, 7,8 ), with FCT measurements accurate to within $13 \pm 9 \%$ on average compared to histology, with which they were strongly correlated (Figure 5). This was also true of plaques with a mean FCT of less than the $650 \mu \mathrm{m}$ threshold for discriminating symptomatic from asymptomatic carotid artery stenoses, as identified by IVUS [13]. The implication is that interventional $3 \mathrm{~T}$ CMR with a loopless guidewire detector could potentially do the same.

Degrading the CMR spatial resolution negatively affected the accuracy of FCT measurements. Increasing the voxel size from $80 \mu \mathrm{m}$ to $140 \mu \mathrm{m}$ resulted in an overestimate of $360 \mu \mathrm{m}$ being recorded for a plaque with a $186 \mu \mathrm{m}$ FCT measured by histology, possible distortions introduced during histological preparation notwithstanding. That an $180 \mu \mathrm{m}$ cap is even observable with a $140 \mu \mathrm{m}$ voxel size attests to the high contrast sensitivity of CMR, and again supports its potential for plaque detection at a coarser but more time-efficient spatial resolution, followed by high-resolution CMR for plaque characterization. Note 

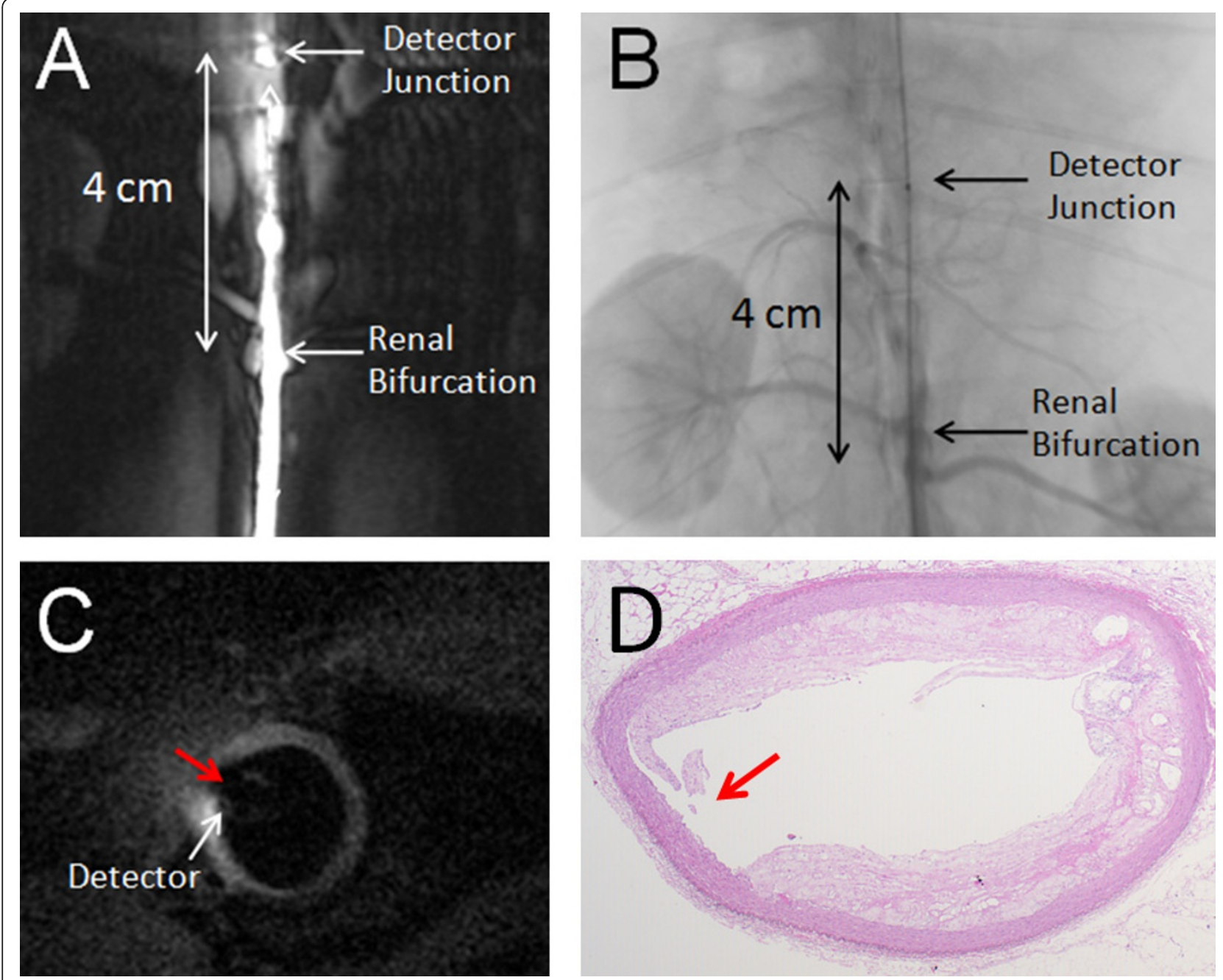

Figure 7 (A) In vivo $\mathrm{T}_{1}$-weighted angiographic coronal image acquired by loopless intravascular CMR detector from a female New Zealand white rabbit showing the aorta near the renal bifurcation. (B) X-Ray fluoroscopic image confirming placement of the loopless CMR detector in the aorta $4 \mathrm{~cm}$ above the renal bifurcation. (C) The cardiac-gated $80 \mu \mathrm{m}$ high-resolution $3 \mathrm{~T}$ CMR of the rabbit aortic vessel wall 1 $\mathrm{cm}$ above the renal bifurcation. (D) Post-mortem histological image of aortic vessel wall of the same imaging slice. The shape is distorted during harvesting and staining processes. A small indent is evident on the vessel wall in both the image (C) and histology (D; red arrows)

also that the averaging of many measurements of FCT from high-resolution CMR can result in reasonably accurate estimates of FCT right down to the native resolution of the scans (Figure 5).

Nevertheless, the $80 \mu \mathrm{m}$ resolution employed here is higher than the $65 \mu \mathrm{m}$ FCT proposed for classifying fibrous caps in coronary plaques as vulnerable [7-10]. In the context of other intravascular imaging modalities, the CMR resolution achieved here is comparable to the best achieved by IVUS at around $100 \mu \mathrm{m}$ [9,11-13]. However, as shown in prior studies comparing CMR with IVUS [14,29], CMR has better soft-tissue contrast and is not limited by the presence of calcifications $[14,29]$, which were common in our sample cohort (eg, Figure 3). We could not match OCT's resolution of $10-20 \mu \mathrm{m}[9,10]$, but CMR's FOV or penetration depth was a good order-of-magnitude larger than in OCT. Also, unlike either of these modalities, intravascular CMR did not require X-ray fluoroscopic guidance. Our scan-times for high-resolution CMR in the aorta were exacerbated by the use of cardiac-gated acquisition. Intravascular CMR of vessels further from the heart might benefit from more-efficient steady-state sequences if gating could be omitted. Also, the promise of a quadratically increasing SNR afforded by even higher $\mathrm{B}_{0}$ human MR scanners [33] offers further hope for $65 \mu \mathrm{m}$ FCT measurements and/or much shorter scan-times.

\section{Conclusion}

In conclusion, the purpose of the present work was to establish the feasibility of $3 \mathrm{~T}$ intravascular CMR for 

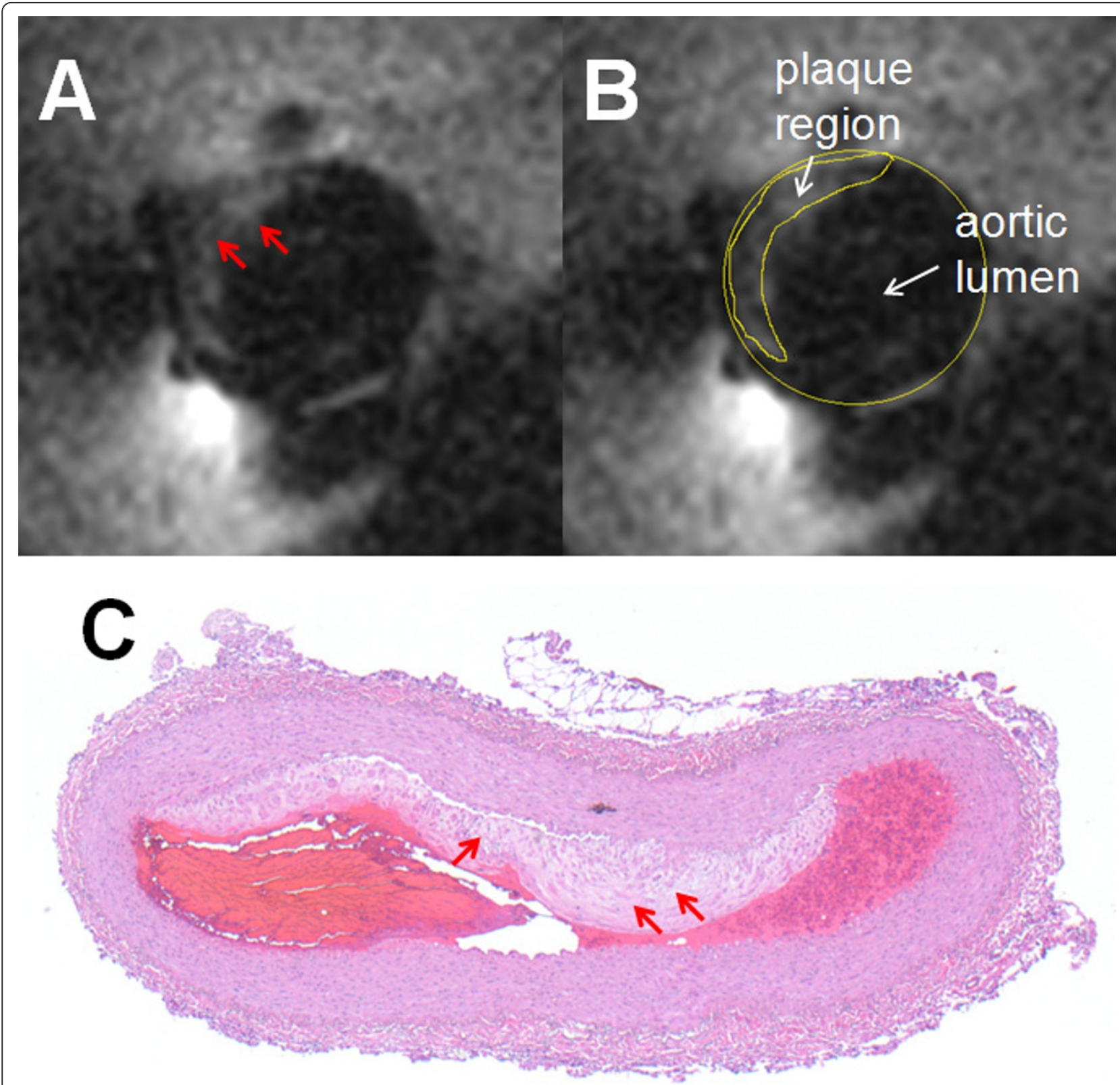

Figure 8 (A) In vivo cardiac-gated $\mathrm{T}_{1}$-weighted $80 \mu \mathrm{m}$ resolution axial $3 \mathrm{~T}$ image acquired from a male hyperlipidemic rabbit aorta showing an area of abnormal tissue extending from the inner tissue wall (red arrows), $1 \mathrm{~cm}$ above the renal bifurcation. (B) The plaque region outlined from the vessel cross-section for quantification. (C) Histology of the corresponding vessel cross-section (collapsed during processing) showing an atherosclerotic lesion (arrows) on the inner wall.

quantifying FCT and to develop a strategy for its use in vivo. We found that a $3 \mathrm{~T}$ loopless intravascular CMR detector could locate atherosclerotic lesions in human arterial specimens in vitro, accurately measure FCT at high-resolution over a range of about 100-1000 $\mu \mathrm{m}$, and that the same approach is feasible in vivo. While it is hoped that $3 \mathrm{~T}$ intravascular CMR may provide a useful imaging option for quantitatively assessing vulnerable plaque and evaluating the efficacy of therapies designed to reduce plaque burden, further work will be required to determine its role in the context of existing intravascular imaging approaches.

\section{Abbreviations}

CMR: cardiovascular magnetic resonance; FCT: fibrous cap thickness; SNR: signal to noise ratio; FOV: field of view; $T_{1}$ : spin-lattice relaxation time; $T_{2}$ : 
spin-spin relaxation time; H\&E: hematoxylin and eosin (stain); OD: outer diameter; 2D: 3D: two: three dimensional; TSE: turbo spin-echo; FFE: fast field echo; TR: repetition time; TE: echo time; FA: flip-angle; IVUS: intravascular ultrasound; OCT: optical coherence tomography; RF: radio frequency.

\section{Acknowledgements}

We thank Lauri Pipitone, BS, for help with animal studies, and Ye Qiao, MD, $\mathrm{PhD}$, for help with the histology. This work was supported by NIH grant R01 HL090728.

\section{Author details}

'Division of MR Research, Department of Radiology and Radiological Science, Johns Hopkins University, Baltimore, MD, USA. ²Division of MR Research, Department of Radiology and Radiological Science, Johns Hopkins School of Medicine, 600 N Wolfe St, Park 310, Baltimore, MD, USA.

\section{Authors' contributions}

$\mathrm{DQ}$ and PAB both contributed to the concept, design, data interpretation, and the drafting and revision of the manuscript. DQ fabricated the experimental devices, conducted the experiments and collected the data. Both authors have read and approved the final manuscript.

\section{Competing interests}

PAB has a financial relationship with MRI-Interventions, Inc (formerly SurgiVision, Inc) from whom the obsolete Intercept 1.5T guidewire was obtained. The relationship is managed in accordance with Johns Hopkins Office of Policy Coordination.

\section{Received: 1 December 2011 Accepted: 26 March 2012} Published: 26 March 2012

\section{References}

1. Ross R. Atherosclerosis-an inflammatory disease. N Engl J Med. 1999;340 (2):115-26. doi:10.1056/NEJM199901143400207.

2. Stary $H C$, Chandler $A B$, Dinsmore RE, et al. A definition of advanced types of atherosclerotic lesions and a histologic classification of atherosclerosis. A report from the Committee on Vascular Lesions of the Council on Arteriosclerosis. American Heart Association Circulation. 1995;92:1355-74.

3. Fuster V, Moreno PR, Fayad ZA, Corti R, Badimon JJ. Atherothrombosis and high-risk plaque: part I: evolving concepts. J Am Coll Cardiol. 2005:46:937-54. doi:10.1016/j.jacc.2005.03.074.

4. Fowkes FG, Housley E, Cawood EH, Macintyre CC, Ruckley CV, Prescott RJ. Edinburgh Artery Study: prevalence of asymptomatic and symptomatic peripheral arterial disease in the general population. Int J Epidemiol. 1991;20:384-92. doi:10.1093/ije/20.2.384.

5. Fuster V, Fayad ZA, Moreno PR, Poon M, Corti R, Badimon JJ. Atherothrombosis and high-risk plaque part II: approaches by noninvasive computed tomographic/magnetic resonance imaging. J Am Coll Cardiol. 2005:46:1 209-18. doi:10.1016/j.jacc.2005.03.075.

6. Li ZY, Howarth SP, Tang T, Gillard JH. How critical is fibrous cap thickness to carotid plaque stability? A flow-plaque interaction model. Stroke. 2006;37:1195-9. doi:10.1161/01.STR.0000217331.61083.3b.

7. Burke AP, Farb A, Malcom GT, Liang YH, Smialek J, Virmani R. Coronary risk factors and plaque morphology in men with coronary disease who died suddenly. N Engl J Med. 1997:336(18):1276-82. doi:10.1056/ NEJM199705013361802.

8. Vengrenyuk $Y$, Carlier $S$, Xanthos $S$, Cardoso L, Ganatos P, Virmani R, Einav $S$, Gilchrist L, Weinbaum S. A hypothesis for vulnerable plaque rupture due to stress-induced debonding around cellular microcalcifications in thin fibrous caps. Proc Natl Acad Sci USA. 2006;103:14678-83. doi:10.1073/ pnas.0606310103.

9. Jang IK, Bouma BE, Kang DH, Park SJ, Park SW, Seung KB, Choi KB, Shishkov M, Schlendorf K, Pomerantsev E, Houser SL, Aretz HT, Tearney GJ. Visualization of coronary atherosclerotic plaques in patients using optical coherence tomography: comparison with intravascular ultrasound. J Am Coll Cardiol. 2002;39:604-9. doi:10.1016/50735-1097(01)01799-5.

10. Tanaka A, Imanishi T, Kitabata H, et al. Morphology of exertion-triggered plaque rupture in patients with acute coronary syndrome: an optical coherence tomography study. Circulation. 2008;118:2368-73. doi:10.1161/ CIRCULATIONAHA. 108.782540.
11. Guagliumi G, Virmani R. The race to achieve the gold standard in coronary imaging. Rev Esp Cardiol. 2009;62(6):599-2.

12. Nishimura RA, Edwards WD, Warnes CA, Reeder GS, Holmes DR, Tajik AJ, et al. Intravascular ultrasound imaging: in vitro validation and pathologic correlation. J Am Coll Cardiol. 1990;16:145-54. doi:10.1016/0735-1097(90) 90472-2.

13. Meissner OA, Rieger J, Rieber J, Klauss V, Siebert U, Tato F, Pfeifer KJ, Reiser M, Hoffmann U. High-resolution MR imaging of human atherosclerotic femoral arteries in vivo: validation with intravascular ultrasound. J Vasc Interv Radiol. 2004;14:227-31.

14. Devuyst $G$, Karapanayiotides $T$, Ruchat $P$, Pusztaszeri M, Lobrinus JA, Jonasson L, Cuisinaire O, Kalangos A, Despland PA, Thiran JP, Bogousslavsky J. Ultrasound measurement of the fibrous cap in symptomatic and asymptomatic atheromatous carotid plaques. Circulation. 2005;111:2776-82. doi:10.1161/CIRCULATIONAHA.104.483024.

15. Brezinski ME, Tearney GJ, Bouma BE, Izatt JA, Hee MR, Swanson EA, Southern JF, Fujimoto JG. Optical coherence tomography for optical biopsy: properties and demonstration of vascular pathology. Circulation. 1996;93:1206-13.

16. Kume T, Akasaka T, Kawamoto T, Okura H, Watanabe N, Toyota E, Neishi Y, Sukmawan R, Sadahira Y, Yoshida K. Measurement of the thickness of the fibrous cap by optical coherence tomography. Am Heart J. 2006;152(4):755. e1-4.

17. Manning WJ, Li W, Edelman RR. A preliminary report comparing magnetic resonance coronary angiography with conventional angiography. N Engl J Med. 1993;328:828-32. doi:10.1056/NEJM199303253281202.

18. Toussaint JF, LaMuraglia GM, Southern JF, Fuster V, Kantor HL. Magnetic resonance images lipid fibrous, calcified, hemorrhagic, and thrombotic components of human atherosclerosis in vivo. Circulation. 1996;94:932-8.

19. Hatsukami TS, Ross R, Polissar NL, Yuan C. Visualization of fibrous cap thickness and rupture in human atherosclerotic carotid plaque in vivo with high-resolution magnetic resonance imaging. Circulation. 2000;102:959-64.

20. Saam T, Cai J, Ma L, Cai YQ, Ferguson MS, Polissar NL, Hatsukami TS, Yuan C. Comparison of symptomatic and asymptomatic atherosclerotic carotid plaque features with in vivo MR imaging. Radiology. 2006:240:464-72. doi:10.1148/radiol.2402050390.

21. Hurst GC, Hua J, Duerk JL, Cohen AM. Intravascular (catheter) NMR receiver probe: Preliminary design analysis and application to canine iliofemoral imaging. Magn Reson Med. 1992;24:343-57. doi:10.1002/mrm.1910240215.

22. Kandarpa K, Jakab P, Patz S, Schoen FJ, Jolesz FA. Prototype miniature endoluminal MR imaging catheter. J Vasc Intervent Radiology. 1993:4:419-27. doi:10.1016/S1051-0443(93)71891-6.

23. Martin AJ, Henkelman RM. Intravascular MR imaging in a porcine animal model. Magn Reson Med. 1994;32:224-9. doi:10.1002/mrm.1910320211.

24. Atalar E, Bottomley PA, Ocali O, Correia LCL, Kelemen MD, Lima JAC, Zerhouni EA. High resolution intravascular MRI and MRS using a catheter receiver coil. Magn Reson Med. 1996;36:596-5. doi:10.1002/ mrm. 1910360415.

25. Ocali O, Atalar E. Intravascular magnetic resonance imaging using a loopless catheter antenna. Magn Reson Med. 1997;37:112-18. doi:10.1002/ mrm.1910370116.

26. Worthley SG, Helft G, Fuster V, Fayad ZA, Shinnar M, Minkoff LA, Schechter C, Fallon JT, Badimon JJ. A novel nonobstructive intravascular MRI coil: in vivo Imaging of experimental atherosclerosis. Arterioscler Thromb Vasc Biol. 2003;23:346-50. doi:10.1161/01.ATV.0000053183.08854.A4.

27. Zimmermann-Paul GG, Quick HH, Vogt $P$, et al. High-resolution intravascular magnetic resonance imaging monitoring of plaque formation in heritable hyperlipidemic rabbits. Circulation. 1999;99:1054-61.

28. Correia LCL, Atalar E, Kelemen MD, et al. Intravascular magnetic resonance imaging of aortic atherosclerotic plaque composition. Arterioscler Thromb Vasc Biol. 1997;17:3626-32. doi:10.1161/01.ATV.17.12.3626.

29. Larose E, Yeghiazarians Y, Libby P, Yucel EK, Aikawa M, Kacher DF, Aikawa E, Kinlay S, Schoen FJ, Selwyn AP, Ganz P. Characterization of human atherosclerotic plaques by intravascular magnetic resonance imaging. Circulation. 2005:112(15):2324-31. doi:10.1161/ CIRCULATIONAHA.105.538942

30. Hofmann LV, Liddell RP, Eng J, Wasserman BA, Arepally A, Lee DS, Bluemke DA. Human peripheral arteries: feasibility of transvenous intravascular MR imaging of the arterial wall. Radiology. 2005;235(2):617-22. doi:10.1148/radiol.2352040340. 
31. Kramer CM, Narula J. Whither catheter-based intravascular magnetic resonance imaging of atherosclerosis? J Am Coll Cardiol Img. 2010;3:1203-4

32. Donald S, Baim DS, Kwong RY. Is Magnetic resonance image guidance the key to opening chronic total occlusions? Circulation. 2006;113:1053-5. doi:10.1161/CIRCULATIONAHA.105.609263.

33. El-Sharkawy AM, Qian D, Bottomley PA. The performance of interventional loopless MRI antennae at higher magnetic field strengths. Med Phys. 2008:35:1995-6. doi:10.1118/1.2905027.

34. Kumar A, Edelstein WA, Bottomley PA. Noise figure limits for circular loop MR coils. Magn Reson Med. 2009;61:1201-9. doi:10.1002/mrm.21948.

35. Qian D, El-Sharkawy AM, Atalar E, Bottomley PA. Interventional MRI: tapering improves the distal sensitivity of the loopless antenna. Magn Reson Med. 2010;63(3):797-2. doi:10.1002/mrm.22152.

36. Sathyanarayana S, Bottomley PA. MRI endoscopy using intrinsically localized probes. Med Phys. 2009:36:908-9. 37. doi:10.1118/1.3077125.

37. Sathyanarayana S, Schär M, Kraitchman DL, Bottomley PA. Towards real-time intravascular endoscopic MRI. J Am Coll Cardiol Img. 2010;3:1158-1.

doi:10.1186/1532-429X-14-20

Cite this article as: Qian and Bottomley: High-resolution intravascular magnetic resonance quantification of atherosclerotic plaque at 3T.

Journal of Cardiovascular Magnetic Resonance 2012 14:20.

\section{Submit your next manuscript to BioMed Central} and take full advantage of:

- Convenient online submission

- Thorough peer review

- No space constraints or color figure charges

- Immediate publication on acceptance

- Inclusion in PubMed, CAS, Scopus and Google Scholar

- Research which is freely available for redistribution

Submit your manuscript at www.biomedcentral.com/submit 